\title{
Serial proton MR spectroscopy and diffusion tensor imaging in infantile Balo's concentric sclerosis
}

\author{
Steffi F. Dreha-Kulaczewski • Gunther Helms • \\ Peter Dechent • Sabine Hofer • Jutta Gärtner • \\ Jens Frahm
}

Received: 4 June 2008 / Accepted: 7 October 2008 / Published online: 29 October 2008

(C) The Author(s) 2008. This article is published with open access at Springerlink.com

\begin{abstract}
Introduction Proton magnetic resonance spectroscopy (MRS) and diffusion tensor imaging (DTI) yield different parameters for characterizing the evolution of a demyelinating white matter disease. The purpose was to elucidate biochemical and microstructural changes in Balo's concentric sclerosis lesions and to correlate the findings with the clinical course.

Methods Localized short-echo time MRS and DTI were performed over 6 years in a left occipital lesion of a female patient (age at onset 13.8 years) with Balo's concentric sclerosis. A right homonym hemianopsia persisted.

Results Metabolite patterns were in line with initial active demyelination followed by gliosis and partial recovery of neuroaxonal metabolites. Fractional anisotropy and mean diffusivity of tissue water remained severely altered. Fiber tracking confirmed a disruption in the geniculo-calcarine tract as well as involvement of the corpus callosum.
\end{abstract}

S. F. Dreha-Kulaczewski $(\bowtie) \cdot J$. Gärtner

Department of Pediatrics and Pediatric Neurology,

Georg August University,

Robert-Koch-Str. 40,

37075 Göttingen, Germany

e-mail: sdreha@gwdg.de

G. Helms $\cdot$ P. Dechent

MR-Research in Neurology and Psychiatry,

Georg August University,

Göttingen, Germany

S. Hofer · J. Frahm

Biomedizinische NMR Forschungs GmbH am

Max-Planck-Institut für biophysikalische Chemie,

Göttingen, Germany

S. Hofer

Bernstein Center for Computational Neuroscience,

Göttingen, Germany
Conclusion MRS and DTI depict complementary parameters, but DTI seems to correlate better with clinical symptoms.

Keywords Balo's concentric sclerosis · Childhood ·

Magnetic resonance spectroscopy .

Diffusion tensor imaging $\cdot \mathrm{tNAA}$ recovery

\section{Introduction}

Balo's concentric sclerosis (BC) is a demyelinating disorder and considered a rare variant of multiple sclerosis (MS) [1]. Pathognomonic MRI features include large concentric ringlike or whorled-appearing lesions in white matter (WM) with alternating hyper- and hypointense bands on T2weighted images with, if active, multiple consecutively enhancing and non-enhancing layers on contrast-enhanced T1-weighted MRI [2]. Neuropathologically, the lesion is characterized by alternating circular lamellae of well preserved and demyelinated WM [3]. Several hypotheses have been brought forward to explain this conspicuous pattern. The myelinated bands have either been considered areas of remyelination that develop between successive episodes of active demyelination [4] or early stages of demyelination [5]. Moore et al. [6] postulated a lesion to start as a confluent area of demyelination which spreads centrifugally, becomes downregulated and blazes up again at the periphery. Immunopathological studies suggested a resemblance to hypoxic tissue injuries as Stadelmann et al. [7] reported signs of neuroprotection at the edge of actively demyelinating bands possibly including oligodendrocytes that are resistant to further damage hence leading to the concentric preservation.

So far, proton magnetic resonance spectroscopy (MRS) studies of $\mathrm{BC}$ have only been performed in adult patients 
$[2,8,9]$, including follow-up measurements over up to 23 months [10-13]. More recently, diffusion tensor imaging (DTI) has been applied [11, 13-15]. However the MRI findings of a 4-year-old boy by Murakami et al. [16] seem to be the only study from the pediatric patient population. Here, we report a combined follow-up MRS and DTI study over almost 6 years of a child diagnosed with BC. The study was carried out to better elucidate underlying biochemical and microstructural alterations depicted by the different MR-methods and their correlations with the evolution of clinical symptoms.

\section{Materials and methods}

\section{Clinical history}

The previously healthy 13.8-year-old girl was admitted at another hospital with an acute right homonym hemianopsia, increasing sleepiness, anomia, and perturbed consciousness following a febrile upper respiratory tract infection. Despite several therapeutic trials (dexamethason, aciclovir, rocephin intravenous and predison per os) the clinical condition worsened. MRI examinations within the first month after onset of symptoms showed a small lesion in left frontal WM with contrast enhancement, another small lesion within right frontal $\mathrm{WM}$, and a most prominent lesion with widespread edema in the left hemisphere involving parietal, temporal, and occipital lobe and demonstrating a temporary cockade-like contrast enhancement.

On admission to our department 1 month later, clinical examination revealed dysarthria, akalulia, sensoric aphasia, and alexia. Further consecutive MRI examinations showed a regression of the pronounced left parieto-occipital (LPO) WM lesion as depicted in Fig. 1a,b (2 months after onset of symptoms) versus Fig. 1c,d (one month later). Cerebrospinal fluid investigations revealed a pleozytosis of 30 cells $/ \mu 1$, oligoclonal $\mathrm{IgG}$, and DNA of human herpes
Fig. 1 MRI of a 13-year-old child with Balo's concentric sclerosis at (a,b) 2 months and (c,d) 3 months after onset of symptoms. a Axial FLAIR image demonstrating the concentric pattern of the two left-hemispheric lesions and $\mathbf{b}$ T1-weighted image depicting the VOI selected for proton MRS. c Axial FLAIR and d T1-weighted image showing regression of the left parietooccipital lesion
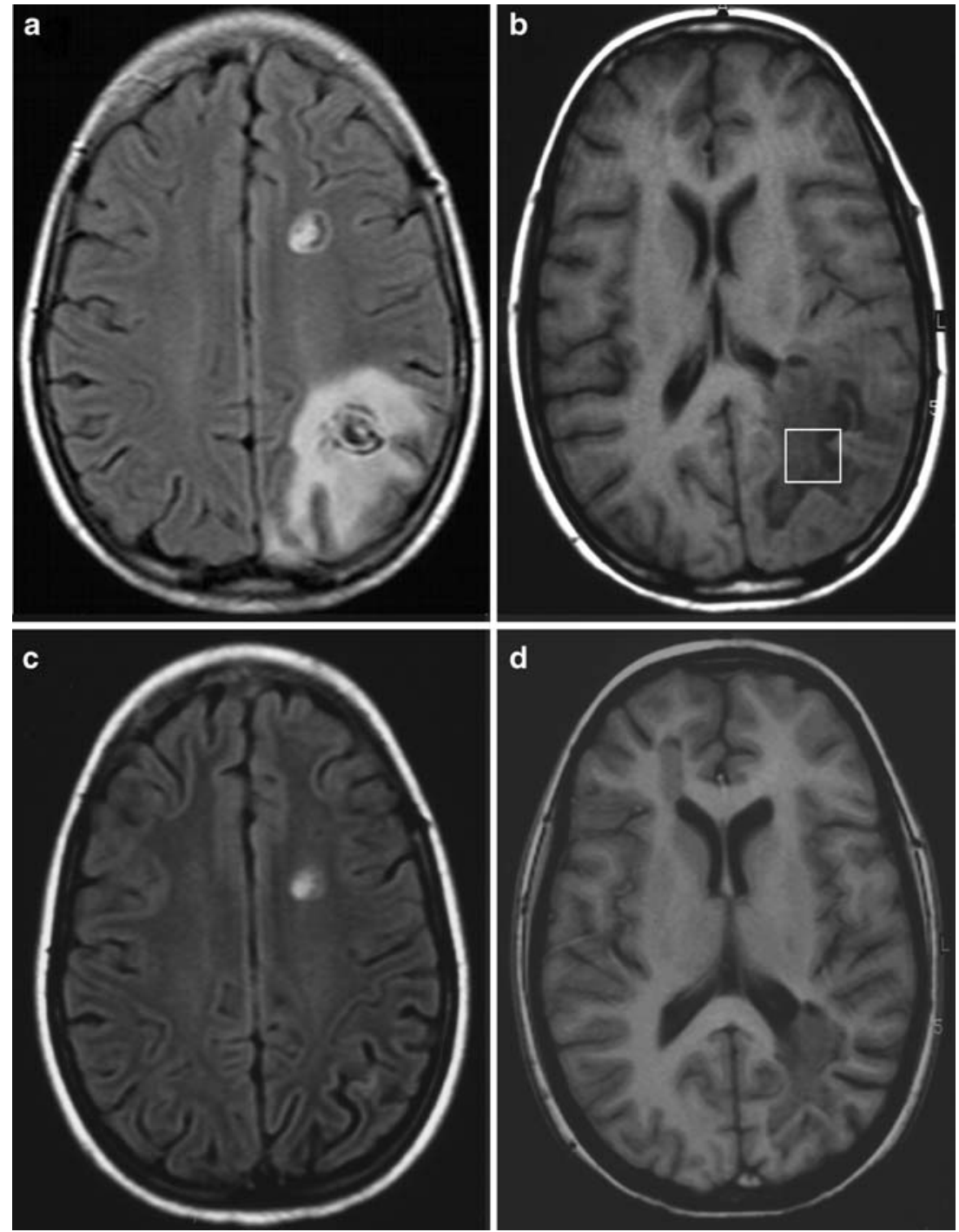
virus type 6 (HHV-6) which lead to an intravenous therapy with foscarnet. In a second spinal tap and serum sample after 4 weeks, no HHV-6 DNA was detectable anymore [17]. Over the following 3 months, all symptoms improved significantly except for the hemianopsia which persists up to this day.

Seven months later, a first relapse occurred with a complex-partial seizure, abulia, and hypokinesia with subsequent remittance. MRI of the brain showed a new contrast-enhancing, concentric lesion in the right frontal premotor region. The known LPO WM lesion appeared smaller. Foscarnet and methylprednisolone were administered intravenously. A maintenance therapy with immunoglobulin ( $2 \mathrm{~g} / \mathrm{kg}$ intravenous) was added for 8 months.

The second relapse occurred 3.4 years after the onset with a left hemiparesis, which was again treated with intravenous methylprednisolone. A new concentric lesion was identified within the right suprathalamic region. On T1-weighted images, the LPO WM lesion had transformed to a hypointense, chronic lesion. The remission has so far been incomplete leaving the patient with a left spastic hemiparesis. She now receives an immunomodulatory therapy with $\alpha$ interferon. Follow-up MRI did not show any new lesion but a considerable atrophy in the LPO WM region.

\section{Proton MR spectroscopy}

A total of seven localized proton MRS studies were performed 1,2 , and 4 months as well as 3.4, 3.8, 4.8, and 5.8 years after the onset of symptoms. The institutional review board approved the study and the parents gave informed consent before each examination. The first three studies were conducted at $2 \mathrm{~T}$ (Magnetom Vision; Siemens Medical Solutions, Erlangen, Germany), the latter four at $3 \mathrm{~T}$ (Magnetom Trio). Proton MR spectra (64 accumulations) were acquired with use of a STEAM localization sequence with repetition time $(\mathrm{TR}) /$ echo time $(\mathrm{TE}) /$ mixing time $(\mathrm{TM})=$ $6000 / 20 / 10 \mathrm{~ms}$ as described $[18,19]$. The $4.85 \mathrm{ml} \mathrm{(2} \mathrm{T)}$ and $4.1 \mathrm{ml}(3 \mathrm{~T})$ volume-of-interest (VOI) was placed within the LPO WM covering the structural lesion but excluding the perifocal edema if possible (indicated in Fig. 1b). For the last MRS examination the VOI was reduced to $2.7 \mathrm{ml}$ to account for tissue loss. Absolute concentrations of $\mathrm{N}$-acetylaspartate and $\mathrm{N}$-acetylaspartylglutamate (tNAA), creatine and phosphocreatine ( $\mathrm{tCr}$ ), choline-containing compounds (Cho), myo-inositol (Ins), and lactate (Lac) were determined by LCModel fitting a linear combination of model spectra [20] and compared to age-matched controls $(n=14)$ taken from Pouwels et al. [21].

\section{Diffusion tensor imaging}

DTI was performed on axial slices of $6 \mathrm{~mm}$ thickness at $2 \mathrm{~T}$ and $4 \mathrm{~mm}$ at $3 \mathrm{~T}$ using a diffusion-weighted partial Fourier single-shot STEAM MRI sequence with $\mathrm{TR}=6.7 \mathrm{~s}$, TE= $74 \mathrm{~ms}(2 \mathrm{~T})$ and $\mathrm{TR}=16.2 \mathrm{~s}, \mathrm{TE}=50 \mathrm{~ms}(3 \mathrm{~T})$, and readout flip angle $10^{\circ}[22,23]$. The protocol comprised a reference image without diffusion-weighting and diffusion-weighted images at a $b$ value of $1000 \mathrm{~s} \mathrm{~mm}^{-2}$ along 24 directions of alternate polarity (icosahedron scheme). Maps of the fractional anisotropy (FA) and mean diffusivity (MD) were calculated from the estimated diffusion tensor and coregistered to anatomical images using FSL 3.2 (FMRIB Software Library, Oxford, U.K.). Regional mean FA and MD values were determined for the same LPO WM lesion as studied by proton MRS (indicated in Fig. 2a). These values were compared to data from age- and gender-matched controls (seven healthy female subjects, age range 1320 years, mean \pm standard deviation (SD) $16.2 \pm 2.9$ years).

To allow for adequate fiber tractography, DTI in the 6th examination was performed at $2.2 \mathrm{~mm}$ isotropic resolution (38 contiguous slices, 3 averages). Estimates of axonal projections were computed by the fiber assignment by continuous tracking (FACT) algorithm using software developed in-house $[24,25]$. Tracking terminated when the FA value was lower than 0.15 or the main diffusion directions in consecutive steps differed by more than $40^{\circ}$ (empirically optimized thresholds). The geniculo-calcarine tracts were determined by region-to-region tracking using regions-of-interest (ROIs) defined on color-coded maps of the main diffusion direction. In order to study secondary degenerative effects of the corpus callosum (CC), the surface of the CC was reconstructed. To compare CC abnormalities of the patient with an age-matched control, a recently developed $\mathrm{CC}$ scheme was applied to delineate the fiber topography of the $\mathrm{CC}$ by separating transcallosal projections into prefrontal, premotor (and supplementary motor), primary motor, primary sensory, parietal, temporal, and occipital regions [26].

\section{Results}

Figure 3 illustrates the time course of metabolic disturbances depicted during follow-up proton MRS of the large LPO WM lesion. Table 1 summarizes corresponding absolute metabolite concentrations in comparison to age-matched controls [21]. The serial metabolite patterns indicated a triphasic development comprising an acute phase 1 month after the onset of symptoms, an intermediate phase extending for at least 3 months, and a chronic phase covering the entire observation period of almost 6 years.

The acute phase was characterized by a reduction of most metabolite concentrations in conjunction with a pronounced elevation of Lac. In more detail, the tNAA concentration was significantly reduced yielding only $22 \%$ of control values. In the intermediate phase, the tNAA level 

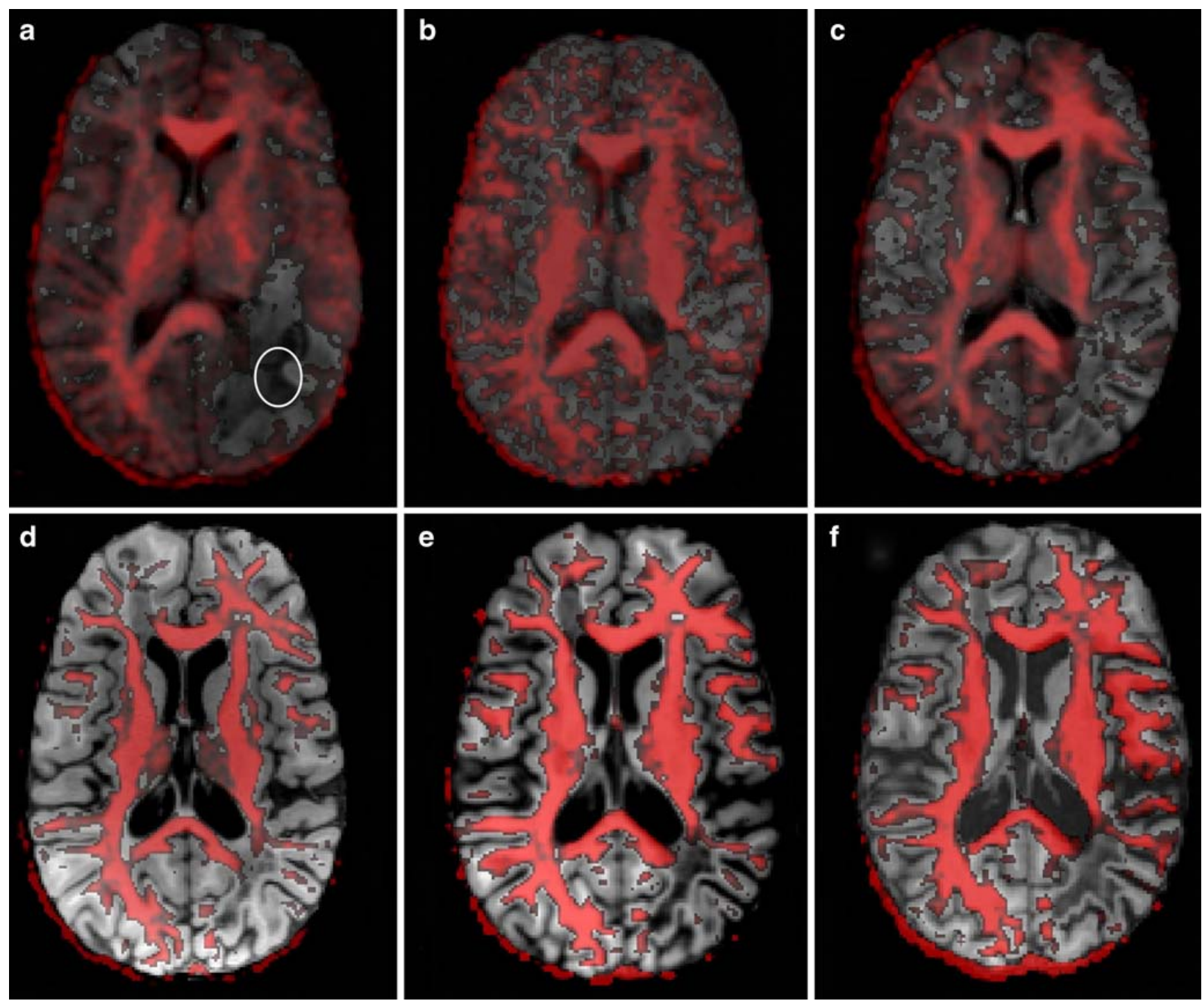

Fig. 2 Thresholded axial fractional anisotropy maps overlaid onto corresponding T1-weighted images at a 1 month, b 2 months, c 4 months, d 3.4 years, e 4.8 years, and $\mathbf{f} 5.8$ years after the onset of symptoms. The ellipse shown in a indicates the location for ROIanalysis of FA and MD values

DTI resulted in reduced anisotropy measures and increased water diffusivities. Figure 2 displays an overlay of FA maps onto T1-weighted images that reveal the loss of anisotropy within the LPO WM region as compared to the contralateral hemisphere. The lesion had its largest extension 1 month after the onset of symptoms (Fig. 2a) and appeared diminished in size already one month later (Fig. 2b). On all subsequent measurements, no further changes could be detected leaving a pronounced lack of anisotropy in the affected area (Fig. 2c-f). Quantitative analyses in Table 2 showed very low FA values of about 0.11 (on a scale from 0 to 1.0) consistent with (almost) isotropic conditions that persisted throughout the entire observation period. The corresponding MD values were significantly elevated in the acute phase (145\% of normal controls) and further 

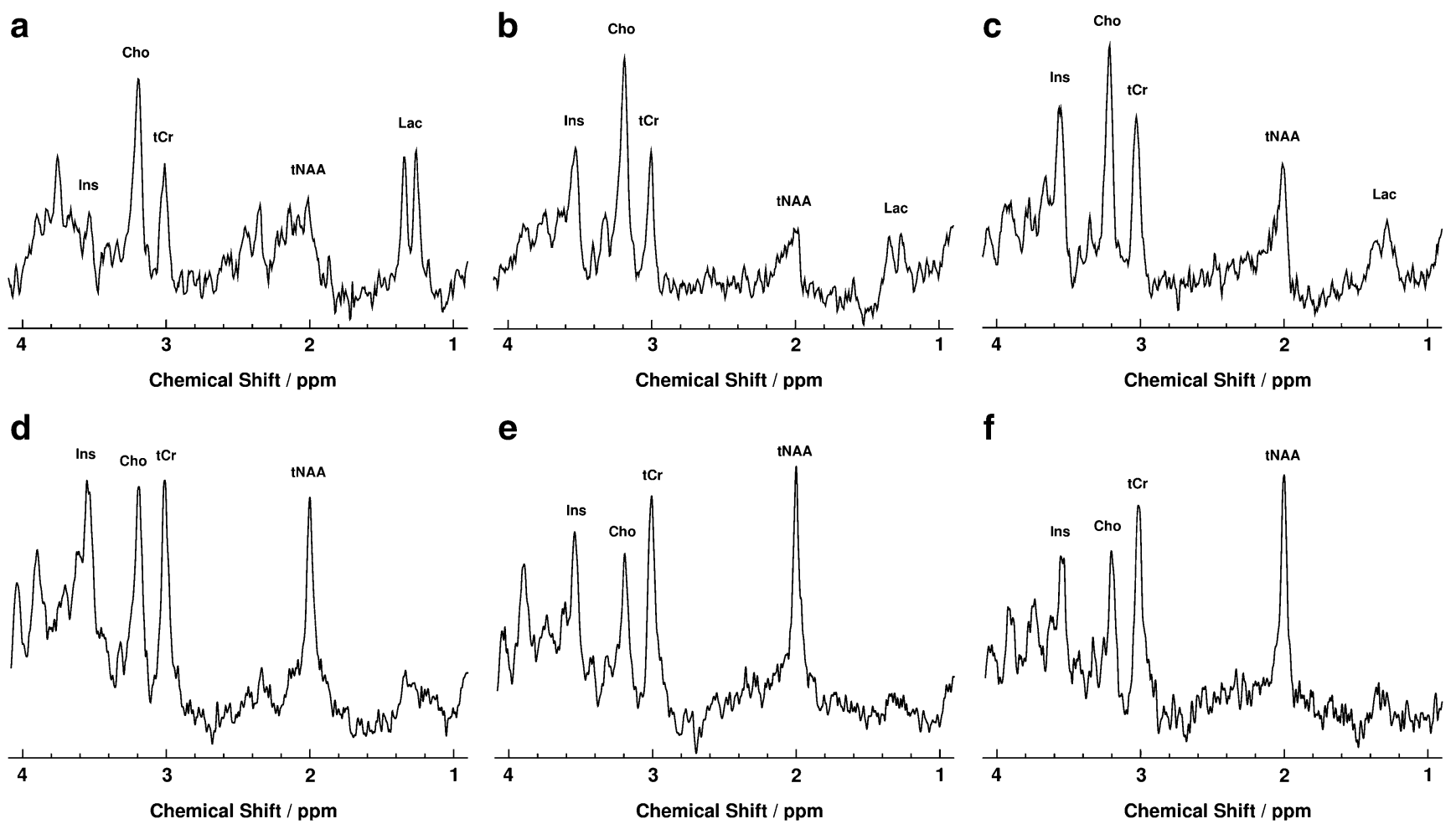

Fig. 3 Serial proton MRS of the left parieto-occipital lesion shown in Fig. 1 at a 1 month, b 2 months, $\mathbf{c} 4$ months, $\mathbf{d} 3.4$ years, e 4.8 years, and $\mathbf{f}$ 5.8 years after the onset of symptoms

increased to a stable level above $200 \%$ of control values in the chronic phase.

The FA maps expose a thinning of the inferior frontooccipital fasciculus and the inferior longitudinal fasciculus as compared to the contralateral side. Fiber tractography revealed a thinning of the ipsilateral geniculo-calcarine tract. As demonstrated in Fig. 4 using three-dimensional views of fiber reconstructions relative to T1-weighted MRI anatomy, tracking of the optic radiation discontinued abruptly at the anterior border of the lesion.

In comparison to an age-matched control, the $\mathrm{CC}$ in Fig. 5 showed a decrease of the $\mathrm{CC}$ volume as constituted by all transcallosal fibers projecting into defined cortical areas (Fig. 5a). The thinning was accompanied by a reduced anisotropy and turned out to be most pronounced in the premotor and supplementary motor area and to a lesser extent in the primary motor cortex (Fig. 5b) which correspond - according to the topographic scheme suggested by Hofer and Frahm [26] - to regions II and III, respectively.

\section{Discussion}

The combined neurochemical and microstructural alterations observed here for a typical BC lesion in vivo are in close agreement with previous findings from histopathological assessments [3-7]. The temporal evolution of the metabolic disturbances is indicative of initial neuroaxonal damage and active demyelination which are followed by partial recovery and gliotic processes. The persistently

Table 1 Absolute metabolite concentrations in the left parieto-occipital white matter lesion.

\begin{tabular}{|c|c|c|c|c|c|c|c|c|}
\hline Time after onset (age) & 1 mo (13.8) & $2 \operatorname{mos}(13.9)$ & $4 \operatorname{mos}(14.1)$ & 3.4 yrs (17.1) & 3.8 yrs (17.4) & 4.8 yrs (18.4) & 5.8 yrs (19.4) & Controls \\
\hline tNAA & $1.7^{\mathrm{a}}$ & $2.8^{\mathrm{a}}$ & $3.8^{\mathrm{a}}$ & $5.0^{\mathrm{a}}$ & $5.2^{\mathrm{a}}$ & $5.6^{\mathrm{a}}$ & $5.4^{\mathrm{a}}$ & $7.9 \pm 0.5$ \\
\hline $\mathrm{tCr}$ & $3.6^{\mathrm{a}}$ & 4.1 & 5.4 & 4.9 & 5.0 & 5.2 & 4.1 & $4.7 \pm 0.5$ \\
\hline Cho & $2.3^{\mathrm{a}}$ & $3.0^{\mathrm{a}}$ & $2.5^{\mathrm{a}}$ & $2.2^{\mathrm{a}}$ & 1.7 & 1.6 & 1.4 & $1.6 \pm 0.2$ \\
\hline Ins & 2.5 & $6.8^{\mathrm{a}}$ & $8.0^{\mathrm{a}}$ & $8.0^{\mathrm{a}}$ & $6.4^{\mathrm{a}}$ & $6.1^{\mathrm{a}}$ & 5.3 & $3.9 \pm 1.1$ \\
\hline $\mathrm{Lac}$ & 8.0 & 4.1 & 4.1 & 1.5 & 1.4 & $<1$ & $<1$ & $<1$ \\
\hline
\end{tabular}

Values are given in $\mathrm{mmol} / \mathrm{l}$ as a function of time after onset of symptoms (yrs years, mo month, mos months) as well as the age (in years) and compared to age-matched controls (mean $\pm \mathrm{SD}, n=14$, age $15.9 \pm 3.5 \mathrm{yrs}$ ) taken from Pouwels et al. [21].

${ }^{a}$ Values differ from controls by $\geq 2 \mathrm{SD}$. 
Table 2 Fractional anisotropy and mean diffusivity in the left parieto-occipital white matter lesion.

Time after onset (age) 1 mo (13.8) 2 mos (13.9) 4 mos (14.1) 3.4 yrs (17.1) 3.8 yrs (17.4) $\quad 4.8$ yrs (18.4) $\quad 5.8$ yrs (19.4) $\quad$ Controls

\begin{tabular}{|c|c|c|c|c|c|c|c|c|}
\hline FA & $0.11^{\mathrm{a}}$ & $0.18^{\mathrm{a}}$ & $0.14^{\mathrm{a}}$ & $0.12^{\mathrm{a}}$ & $0.11^{\mathrm{a}}$ & $0.07^{\mathrm{a}}$ & $0.08^{\mathrm{a}}$ & $0.44 \pm 0.08$ \\
\hline MD & $1.1^{\mathrm{a}}$ & $1.06^{\mathrm{a}}$ & $1.29^{\mathrm{a}}$ & $1.68^{\mathrm{a}}$ & $1.58^{\mathrm{a}}$ & $1.69^{\mathrm{a}}$ & $1.74^{\mathrm{a}}$ & $0.76 \pm 0.05$ \\
\hline
\end{tabular}

Fractional anisotropies (FA) and mean diffusivities (MD, in $10^{-9} \mathrm{~m}^{2} \mathrm{~s}^{-1}$ ) are given as a function of time after onset of symptoms (yrs years, mo month, mos months) as well as the age (in years) and compared to age-matched controls (mean $\pm \mathrm{SD} ; \mathrm{n}=7$ ).

${ }^{a}$ Values differ from controls by $\geq 2 \mathrm{SD}$.

reduced diffusion anisotropy and enhanced water mobility reflect a permanent damage to the tissue structure. These latter findings refer to the neuroaxonal connectivity in both the lesioned area disrupting the optic radiation unilaterally and the premotor and motor regions of the corpus callosum.

Active demyelination and gliosis

The initial metabolite pattern of the LPO WM lesion with strikingly reduced tNAA and high Cho and Lac concentrations is in line with active demyelination. Almost identical metabolic disturbances have frequently been reported for demyelinating processes in conjunction with various leukodystrophies, for example in children with adrenoleukodystrophy [27]. In this context, the tNAA reduction may be taken as a sign of neuroaxonal damage. This is because tNAA has been shown to be predominantly localized within neurons and axons and validated as an axon-specific marker of cerebral WM [28-30]. Complementary, the MRS-detectable Cho resonance comprises precursor molecules for membrane synthesis as well as the corresponding degradation products and therefore relates to structural integrity [31]. The initial high and for almost 3 years elevated Cho concentration may be attributed to ongoing active demyelination and breakdown of myelin phospholipids. However, increased membrane turnover has also been associated with inflammatory cellular infiltration which is known to occur in $\mathrm{BC}$ and therefore a likely contributor to a high Cho level [7, 32]. The subsequent gradual decrease of Cho probably reflects the cessation of active demyelinating processes which are usually replaced by reactive astrocytosis and gliosis. In fact, the course of the Ins concentration, which has been found in high concentrations in astrocytes [33], is in excellent agreement with this histopathological observation.

The Lac concentration was strongly increased 1 month after disease onset and slowly disappeared over time. In general, this finding may indicate non-oxidative glycolysis during ischemia/hypoxia or disturbances in the respiratory chain. Although active $\mathrm{BC}$ lesions resemble aspects found in early hypoxic/ischemic WM as well as in active pattern III MS lesions [7], these are not satisfactory explanations for our patient. Instead, Lac production may be preferentially due to the activation of macrophages and their non-oxidative glucose utilization, which have been found in abundance in $\mathrm{BC}$ lesions $[6,34]$. The observation of mobile lipids in lesions of adult patients with $\mathrm{BC}[8,10]$ could not be confirmed in our study of a child despite the use of short-echo time proton MRS.
Fig. 4 Fiber tractography of the patient's geniculo-calcarine tract 4.8 years after onset of symptoms in three-dimensional views of fiber reconstructions relative to T1-weighted MRI anatomy: a top view and b,c oblique views of the left and right hemisphere, respectively. Colors correspond to the local mean diffusion direction: red leftright, green anterior-posterior, and blue superior-inferior
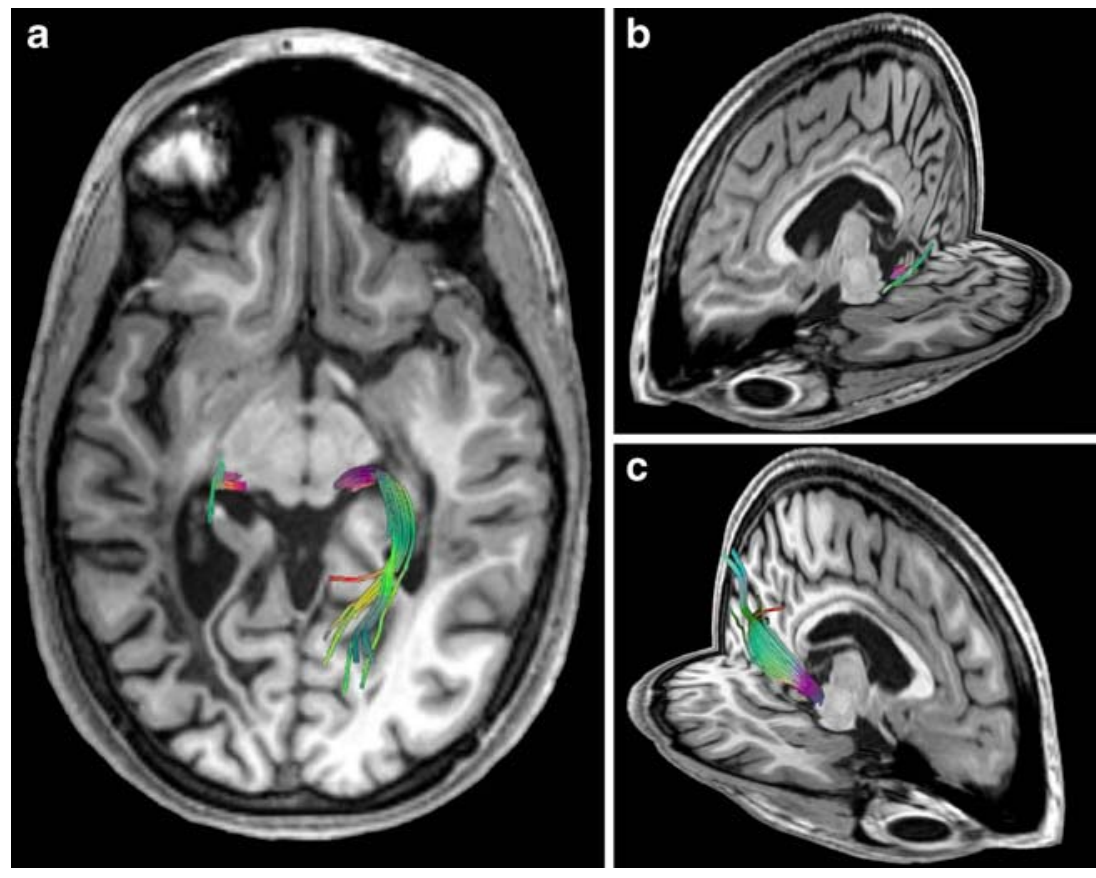
Fig. 5 a,c Sagittal view of $\mathrm{CC}$ surface reconstructions (yellow) and $\mathbf{b}, \mathbf{d}$ corresponding

fractional anisotropy maps of the patient $(\mathbf{a}, \mathbf{b})$ and an agematched control $(\mathbf{c}, \mathbf{d})$. The topographic scheme of the $\mathrm{CC}$ [26] identifies regions with fibers projecting into specific cortical areas: I prefrontal lobe, II premotor and supplementary motor cortex, III motor cortex, $I V$ sensory cortex, $V$ parietal, temporal, and occipital lobes
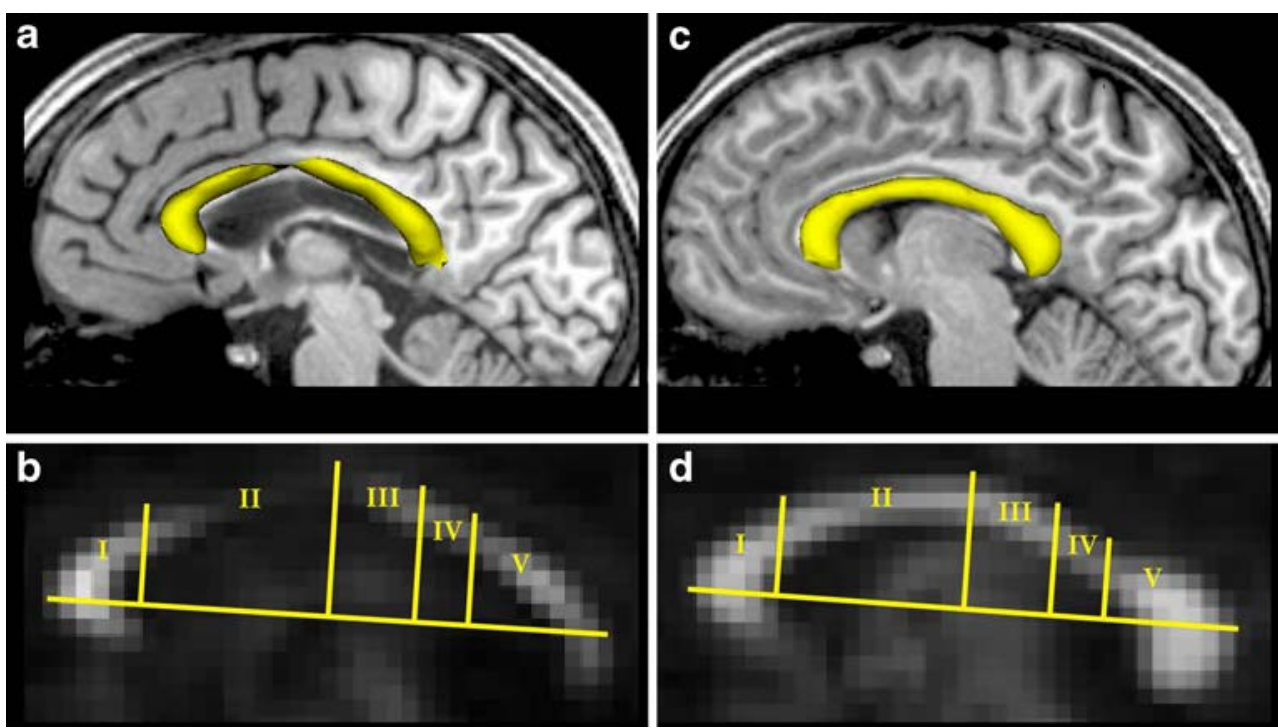

Neuroaxonal damage and recovery

A most remarkable finding of this study is the substantial recovery of tNAA during disease progression. The synthesis of NAA from L-aspartate and acetyl-CoA catalyzed by $N$-acetyl-transferase takes place in the neuronal mitochondria. A translocase transports it into the cytosolic compartment from where it travels the axons via active axonal transport. As NAA cannot be hydrolyzed in these cells, the molecule participates in an intercompartmental cycle between neurons and oligodendrocytes where it is catabolized by aspartoacylase. It has been postulated that NAA turnover is conducted via molecular water pumps and that it plays a major role in brain osmoregulation [35]. Furthermore, NAA has been suggested to act as a precursor for the neurotransmitter NAAG, as an acetyl donor during myelination, and as a molecule participating in interneuronal and intercellular signaling $[35,36]$.

According to the aforementioned functions, several factors may contribute to the reversible reduction of tNAA. Firstly, edema, as a prominent feature of the acute lesion, may simply reduce the relative number of axons per VOI and later reverse the effect upon absorption [32]. Secondly, owing to the osmoregulatory role of NAA, the dramatic changes in extracellular water content during the formation and resolution of the edema could directly affect the intracellular NAA concentration. In fact, in animal models without loss of neuron viability, NAA was greatly reduced or even absent presumably solely because of its function as a neuronal osmolyte [37]. Thirdly, the mitochondrial function may be reversibly impaired during inflammation in which case NAA synthesis is resumed to a significant extent at later stages [36]. Fourthly, it could be speculated that the axonal transport is re-established after subsidence of active demyelination in parallel to remyelination. The persisting reduction of tNAA to about $70 \%$ of control values can very well be attributed to the residual atrophy, that is permanent neuroaxonal loss, within the LPO WM. This explanation is not only appreciable on MRI but also supported by the structural deficits revealed by DTI.

\section{White matter fiber structure}

WM diffusion anisotropy reflects the integrity of both axonal membranes and myelin sheaths. FA and MD values in our patient were significantly altered in the BC lesion and in contrast to the MRS results showed no recovery. Reduced anisotropy and increased diffusivity point to a structural damage within the delicate microarchitecture of axonal membranes, periaxonal spaces, and myelin layers which apparently remained without repair. These results are in line with data from MS patients where a range of studies document similar changes in response to a structural tissue damage, for example see Rovaris et al. [38]. Two DTI studies of adult BC reported initially reduced rather than enhanced water diffusivity in active lesions [15, 39]. The fact that we did not see such effects one month after disease onset may be due to their use of very early time points as close as day one of the disease. Almost immediate reductions of the apparent diffusion coefficient that several days later are followed by a reversal to higher values than normal are a typical observation in ischemic brain tissue after acute stroke [40].

Fiber tractography has so far not been performed in BC. It revealed a significantly impaired optic radiation in the left hemisphere almost 5 years after disease onset. The abrupt fiber truncation at the anterior border of the lesion is in full accordance with the persisting clinical symptom of right homonym hemianopsia. Neuroaxonal damage together with gliotic changes may be responsible not only for the lack of 
structural integrity and, hence, diffusion anisotropy, but also for impairing normal axonal transport and function. Furthermore, a surface reconstruction of the CC showed a pronounced thinning of fiber regions interconnecting rightand left-hemispheric premotor and supplementary motor areas as well as primary motor cortices [26]. In a previous study, Sydykova et al. reported a correlation between reduced FA values in the $\mathrm{CC}$ and a decline in fiber integrity and neurodegeneration in corresponding cortical areas [41]. Therefore, reduced FA values in CC regions covering fibers of cortical premotor and supplementary motor areas (compare Fig. 5b) fit well to the observed premotor lesions in both hemispheres.

\section{Concluding remarks}

Serial proton MRS and DTI of a child with BC revealed a coherent pattern of neurochemical and structural abnormalities and respective changes during disease progression. Metabolic disturbances were consistent with acute demyelination followed by gliosis and partial recovery of neuroaxonal metabolite concentrations. DTI resulted in persistent alterations of the microstructure not only in the MRI-detectable lesion but also in specific regions of the $\mathrm{CC}$ associated with motor functions.

In conclusion, proton MRS and DTI sensitively depict different processes during the evolution of a $\mathrm{BC}$ lesion. Whereas MRS monitors respective metabolic disturbances, DTI-based structural alterations seem to correlate better with the clinical symptoms. The combination of both methods in a clinical setting allows for a more detailed analysis of lesion development and may contribute to the establishment of prognostic criteria.

Acknowledgments SF D-K was supported by the Bundesministerium für Bildung und Forschung (01GM0309). GH and PD were supported by the VolkswagenStiftung.

Conflict of interest statement We declare that we have no conflict of interest.

Open Access This article is distributed under the terms of the Creative Commons Attribution Noncommercial License which permits any noncommercial use, distribution, and reproduction in any medium, provided the original author(s) and source are credited.

\section{References}

1. Kastrup O, Stude P, Limmroth V (2002) Balo's concentric sclerosis. Evolution of active demyelination demonstrated by serial contrast-enhanced MRI. J Neurol 249:811-814 doi:10.1007/ s00415-002-0718-0

2. Karaarslan E, Altintas A, Senol U et al (2001) Balo's concentric sclerosis: clinical and radiologic features of five cases. AJNR Am J Neuroradiol 22:1362-1367

3. Nandini M, Gourie-Devi M, Shankar SK et al (1993) Balo's concentric sclerosis diagnosed intravitam on brain biopsy. Clin Neurol Neurosurg 95:303-309 doi:10.1016/0303-8467(93)90106Q

4. Moore GR, Neumann PE, Suzuki K et al (1985) Balo's concentric sclerosis: new observations on lesion development. Ann Neurol 17:604-611 doi:10.1002/ana.410170614

5. Yao DL, Webster HD, Hudson LD et al (1994) Concentric sclerosis (Balo): morphometric and in situ hybridization study of lesions in six patients. Ann Neurol 35:18-30 doi:10.1002/ ana.410350105

6. Moore GR, Berry K, Oger JJ et al (2001) Balo's concentric sclerosis: surviving normal myelin in a patient with a relapsingremitting dinical course. Mult Scler 7:375-382

7. Stadelmann C, Ludwin S, Tabira T et al (2005) Tissue preconditioning may explain concentric lesions in Balo's type of multiple sclerosis. Brain 128:979-987 doi:10.1093/brain/ awh457

8. Kim MO, Lee SA, Choi CG et al (1997) Balo's concentric sclerosis: a clinical case study of brain MRI, biopsy, and proton magnetic resonance spectroscopic findings. J Neurol Neurosurg Psychiatry 62:655-658

9. Khiat A, Lesage J, Boulanger Y (2007) Quantitative MRS study of Balo's concentric sclerosis lesions. Magn Reson Imaging 25:1112-1115 doi:10.1016/j.mri.2006.11.005

10. Chen CJ, Chu NS, Lu CS et al (1999) Serial magnetic resonance imaging in patients with Balo's concentric sclerosis: natural history of lesion development. Ann Neurol 46:651-656 doi:10.1002/15318249(199910)46:4<651::AID-ANA15>3.0.CO;2-Y

11. Rovira A, Pericot I, Alonso J et al (2002) Serial diffusionweighted MR imaging and proton MR spectroscopy of acute large demyelinating brain lesions: case report. AJNR Am J Neuroradiol 23:989-994

12. Chen CJ (2001) Serial proton magnetic resonance spectroscopy in lesions of Balo concentric sclerosis. J Comput Assist Tomogr 25:713-718 doi:10.1097/00004728-200109000-00008

13. Lindquist S, Bodammer N, Kaufmann J et al (2007) Histopathology and serial, multimodal magnetic resonance imaging in a multiple sclerosis variant. Mult Scler 13:471-482

14. Ball T, Malik O, Roncaroli F et al (2007) Apparent diffusion coefficient changes and lesion evolution in Balo's type demyelination - correlation with histopathology. Clin Radiol 62:498-503 doi:10.1016/j. crad.2006.11.020

15. Kavanagh EC, Heran MK, Fenton DM et al (2006) Diffusionweighted imaging findings in Balo concentric sclerosis. $\mathrm{Br} \mathrm{J}$ Radiol 79:e28-e31 doi:10.1259/bjr/36636301

16. Murakami Y, Matsuishi T, Shimizu T et al (1998) Balo's concentric sclerosis in a 4-year-old Japanese infant. Brain Dev 20:250-252 doi:10.1016/S0387-7604(98)00025-4

17. Pohl D, Rostasy K, Krone B et al (2005) Balo's concentric sclerosis associated with primary human herpesvirus 6 infection. J Neurol Neurosurg Psychiatry 76:1723-1725 doi:10.1136/ jnnp.2004.062331

18. Frahm J, Bruhn H, Gyngell ML et al (1989) Localized highresolution proton NMR spectroscopy using stimulated echoes: initial applications to human brain in vivo. Magn Reson Med 9:79-93 doi:10.1002/mrm.1910090110

19. Frahm J, Michaelis T, Mehrboldt KD et al (1990) Improvements in localized proton NMR spectroscopy of human brain. Water suppression, short echo times, and $1 \mathrm{ml}$ resolution. J Magn Reson 90:464-473 
20. Provencher SW (1993) Estimation of metabolite concentrations from localized in vivo proton NMR spectra. Magn Reson Med 30:672-679 doi:10.1002/mrm.1910300604

21. Pouwels PJ, Brockmann K, Kruse B et al (1999) Regional age dependence of human brain metabolites from infancy to adulthood as detected by quantitative localized proton MRS. Pediatr Res 46:474-485 doi:10.1203/00006450-199910000-00019

22. Rieseberg S, Merboldt KD, Kuntzel M et al (2005) Diffusion tensor imaging using partial Fourier STEAM MRI with projection onto convex subsets reconstruction. Magn Reson Med 54:486490 doi: $10.1002 / \mathrm{mrm} .20572$

23. Nolte UG, Finsterbusch J, Frahm J (2000) Rapid isotropic diffusion mapping without susceptibility artifacts. Whole brain studies using diffusion-weighted single-shot STEAM MR imaging. Magn Reson Med 44:731-736 doi:10.1002/1522-2594 (200011)44:5<731::AID-MRM11>3.0.CO;2-1

24. Mori S, Crain BJ, Chacko VP et al (1999) Three-dimensional tracking of axonal projections in the brain by magnetic resonance imaging. Ann Neurol 45:265-269 doi:10.1002/1531-8249 (199902)45:2<265::AID-ANA21>3.0.CO;2-3

25. Küntzel M (2007) Parallele Datenakquisition zur Beschleunigung diffusionsgewichteter Kernspintomographie mit stimulierten Echos [Parallel data acquisition for diffusion-weighted MRI using stimulated echoes]. Dissertation, Georg-August-Universität, Göttingen. Online access University library: http://webdoc.sub.gwdg.de/diss/ 2007/kuentzel/kuentzel.pdf.

26. Hofer S, Frahm J (2006) Topography of the human corpus callosum revisited - comprehensive fiber tractography using diffusion tensor magnetic resonance imaging. Neuroimage 32:989994 doi:10.1016/j.neuroimage.2006.05.044

27. Pouwels PJ, Kruse B, Korenke GC et al (1998) Quantitative proton magnetic resonance spectroscopy of childhood adrenoleukodystrophy. Neuropediatrics 29:254-264 doi:10.1055/s-2007973571

28. Bjartmar C, Battistuta J, Terada $\mathrm{N}$ et al (2002) $\mathrm{N}$-acetylaspartate is an axon-specific marker of mature white matter in vivo: a biochemical and immunohistochemical study on the rat optic nerve. Ann Neurol 51:51-58 doi:10.1002/ana.10052

29. Moffett JR, Namboodiri MA, Cangro CB et al (1991) Immunohistochemical localization of $\mathrm{N}$-acetylaspartate in rat brain.
Neuroreport 2:131-134 doi:10.1097/00001756-19910300000005

30. Simmons ML, Frondoza CG, Coyle JT (1991) Immunocytochemical localization of $\mathrm{N}$-acetyl-aspartate with monoclonal antibodies. Neuroscience 45:37-45 doi:10.1016/0306-4522(91)90101-S

31. Frahm J, Hanefeld F (1996) Localized proton magnetic resonance spectroscopy of cerebral metabolites. Neuropediatrics 27:64-69 doi:10.1055/s-2007-973751

32. Davie CA, Hawkins CP, Barker GJ et al (1994) Serial proton magnetic resonance spectroscopy in acute multiple sclerosis lesions. Brain 117(Pt 1):49-58 doi:10.1093/brain/117.1.49

33. Brand A, Richter-Landsberg C, Leibfritz D (1993) Multinuclear NMR studies on the energy metabolism of glial and neuronal cells. Dev Neurosci 15:289-298 doi:10.1159/000111347

34. Lopez-Villegas D, Lenkinski RE, Wehrli SL et al (1995) Lactate production by human monocytes/macrophages determined by proton MR spectroscopy. Magn Reson Med 34:32-38 doi:10.1002/mrm.1910340107

35. Baslow MH (2003) N-acetylaspartate in the vertebrate brain: metabolism and function. Neurochem Res 28:941-953 doi:10.1023/A:1023250721185

36. De Stefano N, Matthews PM, Arnold DL (1995) Reversible decreases in $N$-acetylaspartate after acute brain injury. Magn Reson Med 34:721-727 doi:10.1002/mrm.1910340511

37. Baslow MH, Suckow RF, Gaynor K et al (2003) Brain damage results in down-regulation of $\mathrm{N}$-acetylaspartate as a neuronal osmolyte. Neuromolecular Med 3:95-104 doi:10.1385/NMM:3:2:95

38. Rovaris M, Gass A, Bammer R et al (2005) Diffusion MRI in multiple sclerosis. Neurology 65:1526-1532 doi:10.1212/01. wnl.0000184471.83948.e0

39. Wiendl $H$, Weissert R, Herrlinger $U$ et al (2005) Diffusion abnormality in Balo's concentric sclerosis: clues for the pathogenesis. Eur Neurol 53:42-44 doi:10.1159/000084264

40. Lansberg MG, Thijs VN, O'Brien MW et al (2001) Evolution of apparent diffusion coefficient, diffusion-weighted, and T2-weighted signal intensity of acute stroke. AJNR Am J Neuroradiol 22:637-644

41. Sydykova D, Stahl R, Dietrich O et al (2006) Fiber Connections between the Cerebral Cortex and the Corpus Callosum in Alzheimer's Disease: A Diffusion Tensor Imaging and VoxelBased Morphometry Study. Cereb Cortex 17:2276-2282 